\title{
A small angle $X$-ray scattering study of nanoparticle assembly in an aligned nematic liquid crystal
}

\section{Supplemental Information}

\section{Scanning electron microscopy:}
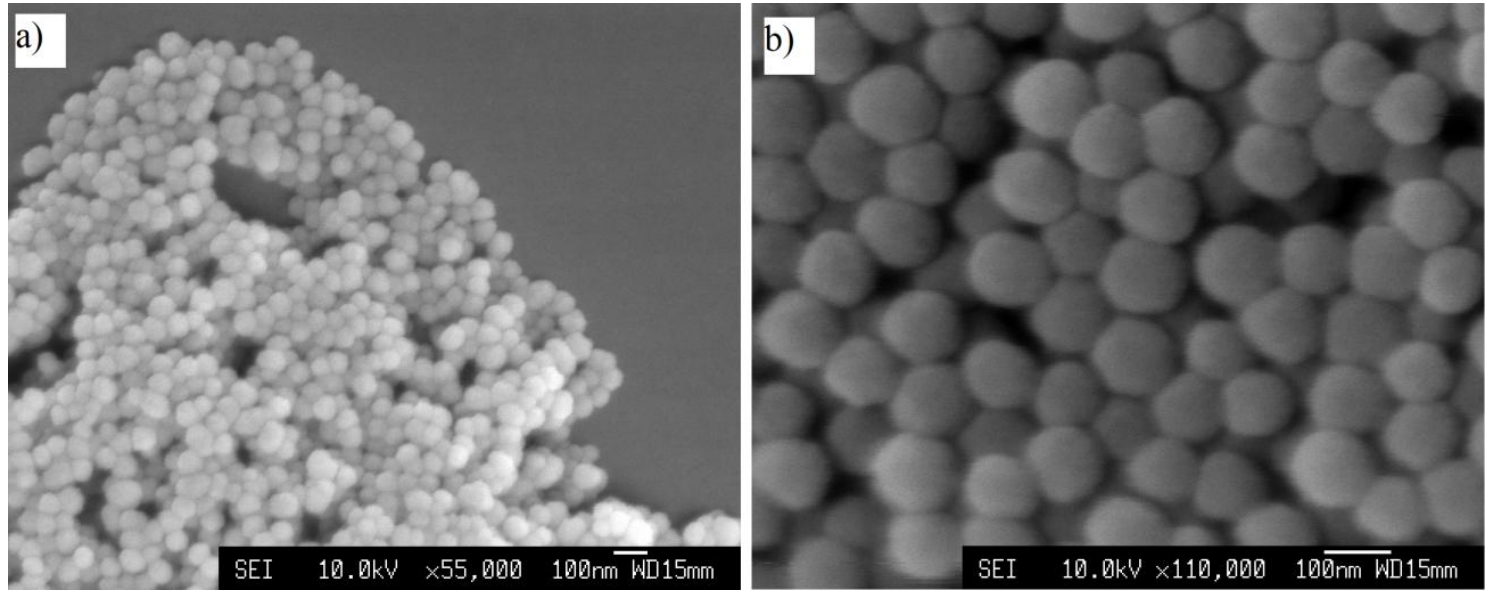

Figure S1: SEM images of silica nanoparticles of approximate size a) $50 \mathrm{~nm}$ and b) $100 \mathrm{~nm}$. The scale bars are $100 \mathrm{~nm}$ in both panels.

The size and shape of the modified silica nanoparticles was also verified using scanning electron microscopy (SEM) using a JEOL JSM 6330F field-emission gun SEM. Films of particles were produced by drop-casting dilute suspensions of particles onto freshly cleaved mica substrates, before sputter coating with gold of approximately $10 \mathrm{~nm}$ thickness for measurement. The particles appeared to be spherical and their diameters were measured and determined to be $114 \mathrm{~nm} \pm 10 \mathrm{~nm}$ and $62 \mathrm{~nm} \pm 8 \mathrm{~nm}$. The disparity between the sizes determined from the different techniques can be attributed to the gold layer deposited on the particles measured with SEM; therefore the sizes and polydispersities determined through SAXS of dilute suspensions in methanol were used throughout. 


\section{SAXS in isotropic solvent:}

Scattering patterns for particles in methanol were obtained using the same method as for those in liquid crystal. The scattering pattern with and without an electric field is identical indicating no structural response to the electric field.


Figure S2: Small angle X-ray scattering patterns for $\eta=0.002,105 \mathrm{~nm}$ DMOAP-modified silica in methanol at a) zero applied field and b) $4 \mathrm{MVm}^{-1}$. 